\title{
The effects of ultrasound on blood-brain barrier
}

\author{
Feng-Yi Yang, Wei-Ting Lin
}

Department of Biomedical Imaging and Radiological Sciences, National Yang-Ming University, Taipei, Taiwan

Email: fyyang@ym.edu.tw, lin_79_03_26@yahoo.com.tw

Received August 2013

\begin{abstract}
The brain is protected from the entry of foreign substances by blood-brain barrier (BBB), but becomes a barrier while chemotherapy is needed for the brain diseases. Ultrasound with microbubbles (MBs) has been shown to noninvasively increase the permeability of the BBB in the normal tissue and brain tumor. The real mechanism for disruption is still unknown. Hemorrhage was usually found in the sonicated region of the brain. Thus, treatment safety is the primary concern when considering clinical application of BBB disruption induced by ultrasound in the presence of MBs. Here we investigate the effects of ultrasound on the permeability of BBB whether the MBs were administered. The data reveals that Evans blue (EB) accumulation was highest in the brain after sonication with MBs. However, the permeability of BBB also can be significantly increased by ultrasound alone. These results demonstrated that noninvasive disruption of BBB by ultrasound alone with no damage is possible.
\end{abstract}

Keywords: Ultrasound; Blood-Brain Barrier; Permeability; Drug Delivery; Brain Disease

\section{INTRODUCTION}

Most brain diseases are hard to treat with chemotherapeutics due to the inability of molecules to pass the blood-brain barrier (BBB). The endothelial cells of the brain are tightly fused to each other known as tight junctions [1]. Several methods have been developed to disrupt the BBB to facilitate drug delivery. Recently, it has been shown that BBB can be locally and noninvasively disrupted by focused ultrasound (FUS) in the presence of microbubbles (MBs) [2-4]. However, small areas of erythrocyte extravasation were found in the sonicated site $[5,6]$. Interactions between the ultrasound and MBs - which include oscillatory forces, acoustic cavitation, and shear stress related to streaming of fluid around the bubbles-are likely to trigger various physiological responses [7]. The side effect of hemorrhage may be induced by the widening of the tight junction or vessel damage after FUS sonication. In this study, we investigate if $\mathrm{BBB}$ can be disrupted noninvasively by FUS without MBs administration in order to avoid the brain damage from cavitation effects.

\section{METHODS}

\subsection{Animal Preparation}

A total of twelve male Sprague-Dawley rats weighing from 280 to $350 \mathrm{~g}$ were used in these experiments. All the procedures of the animal experiment adhered to the Guidelines for Care and Use of Experimental Animals by our institutional animal committee.

\subsection{Ultrasound System}

FUS was produced by a $1 \mathrm{MHz}$ single-element focused transducer (A392S, Panametrics, Waltham, MA, USA) with a diameter of $38 \mathrm{~mm}$ and a radius of curvature of $63.5 \mathrm{~mm}$. The half-maximum of the pressure amplitude of the focal zone had a diameter and length of 3 and 26 $\mathrm{mm}$, respectively. The transducer was mounted on a removable cone filled with deionized and degassed water whose tip was capped by a polyurethane membrane, and the center of the focal spot was at approximately $5.7 \mathrm{~mm}$ below the cone tip. FUS beam was precisely targeted using a stereotaxic apparatus (Stoelting, Wood Dale, IL, USA). A function generator (33220A, Agilent Inc., Palo Alto, USA) was connected to a power amplifier (500-009, Advanced Surgical Systems, Tucson, AZ) to drive the FUS transducer and a power meter/ sensor module (Bird 4421, Ohio, USA) was used to measure the input electrical power. The animal positioning for the sonication arrangement was the same as our previous works $[8,9]$. The rat was laid prone beneath the cone tip and ultrasound transmission gel (Pharmaceutical Innovations, Newark, NJ, USA) was used to maximize the transmission of ultrasound between the transducer and the rat's brain.

\subsection{Soncation}

Ultrasound contrast agent (UCA, SonoVue, Bracco In- 
ternational, Amsterdam, The Netherlands) was injected into the tail vein of the rats about $15 \mathrm{~s}$ before each sonication. This agent contains phospholipid-coated microbubbles at a concentration of $1-5 \times 10^{8}$ bubbles $/ \mathrm{ml}$, with the bubbles having a mean diameter of $2.5 \mu \mathrm{m}$. The sonication was precisely targeted using a stereotaxic apparatus. The ultrasound beam was delivered to one location in one brain hemisphere. The following sonication parameters were used: an acoustic power of $2.86 \mathrm{~W}$ with or without an injection of $300 \mu \mathrm{l} / \mathrm{kg}$ UCA, an acoustic power of $1.01 \mathrm{~W}$ without UCA injection, a pulse repetition frequency of $1 \mathrm{~Hz}$, and a duty cycle of 5\%. The schematic diagram of the FUS system for sonication is shown in Figure 1.

\subsection{Evans Blue Extravasation}

When measuring vascular permeability, it has been shown that BBB disruption can be quantified based on the extravasation of EB. The animals were sacrificed approximately 4 hours after the EB injection. These rats were then perfused with saline via the left ventricle until colorless perfusion fluid appeared from the right atrium. After perfusion and brain removal, the brain was sectioned into four slices (6 $\mathrm{mm}$ posterior to the bregma) and mounted on glass slides. The four sections were then divided into right and left hemispheres before measuring the amount of EB extravasation. The unsonicated hemispheres formed the control groups. Samples were weighed and then soaked in 50\% trichloroacetic acid solution. After homogenization and centrifugation, the extracted dye was diluted with ethanol (1:3), and the amount of dye present measured using a spectrophotometer (PowerWave 340, BioTek, USA) at $620 \mathrm{~nm}$. The EB present in the tissue samples was quantified using a linear regression standard curve derived from seven concentrations of the dye; the amount of dye was denoted in absorbance per gram of tissue. All data are typically expressed as means \pm SEM. Statistical analysis was per-

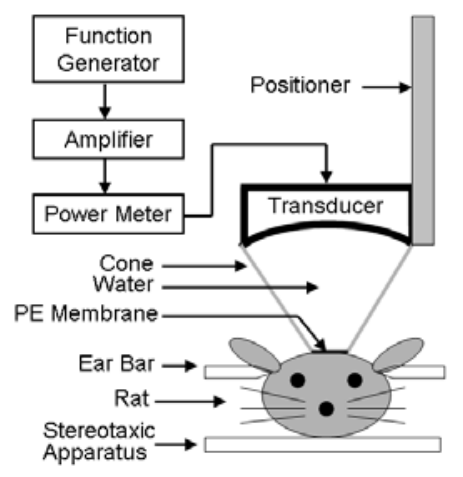

Figure 1. Diagrams of the experimental FUS setup for BBB disruption. formed with a standard $t$-test. Statistical significance was defined as $p$ values $\leq 0.05$.

\section{RESULTS}

Two experimental protocols were performed to assess firstly the degree of the $\mathrm{BBB}$ disruption that occurs at an acoustic power of $2.86 \mathrm{~W}$ with an injection of 300 $\mu \mathrm{l} / \mathrm{kg} \mathrm{MBs}$ and secondly the effect of various sonication powers on the BBB permeability.

\subsection{BBB Disruption by FUS with MBs}

In the first protocol, three rats were sonicated with FUS in the presence of MBs for the sonication time of $1 \mathrm{~min}$. Figure 2 shows that a sonication with MBs is able to significantly increase the accumulation of $\mathrm{EB}$ in the sonicated brain compared to the unsonicated brain.

\subsection{Effect of FUS on BBB Permeability}

Another experiment was performed to investigate the effect of FUS alone on the permeability of BBB at an acoustic power of $2.86 \mathrm{~W}$ or $1.01 \mathrm{~W}$ for the sonication time of $5 \mathrm{~min}$.

In Figure 3, we showed comparisons of EB extravasation in the right or left hemisphere brain after FUS exposure alone at an acoustic power of $2.86 \mathrm{~W}$. No matter right or left hemisphere brain, EB extravasation in the contralateral brain was significantly higher than that in the sonicated brain, especially for the left contralateral brain. In addition, the EB extravasation was similar between the right brain and left brain after FUS exposure.

Oppositely, Figure 4 shows that EB extravasation in the sonicated brain was significantly higher than that in the contralateral brain while a lower acoustic power of $1.01 \mathrm{~W}$ was applied.

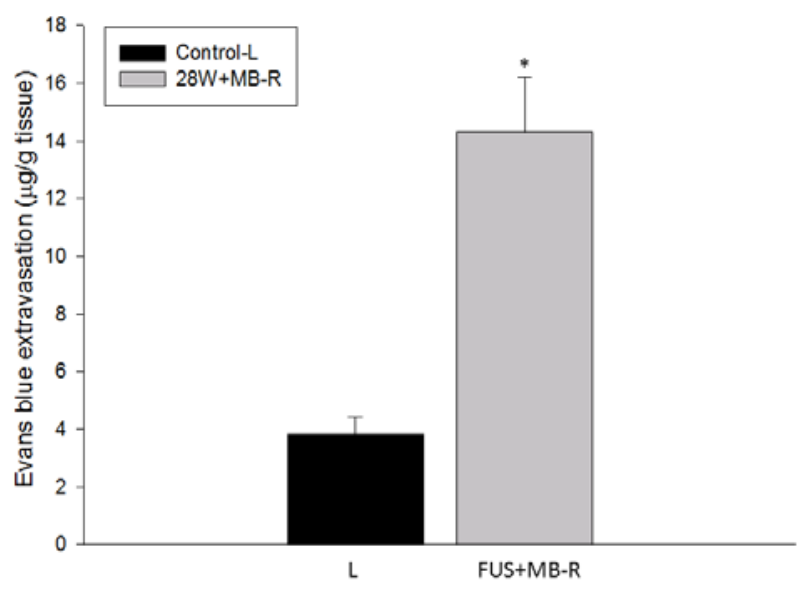

Figure 2. EB extravasation for the brain sonicated at an acoustic power of $2.86 \mathrm{~W}$ with $300 \mu \mathrm{l} / \mathrm{kg}$ UCA. *Denote significant difference compared with the contralateral normal brain. 

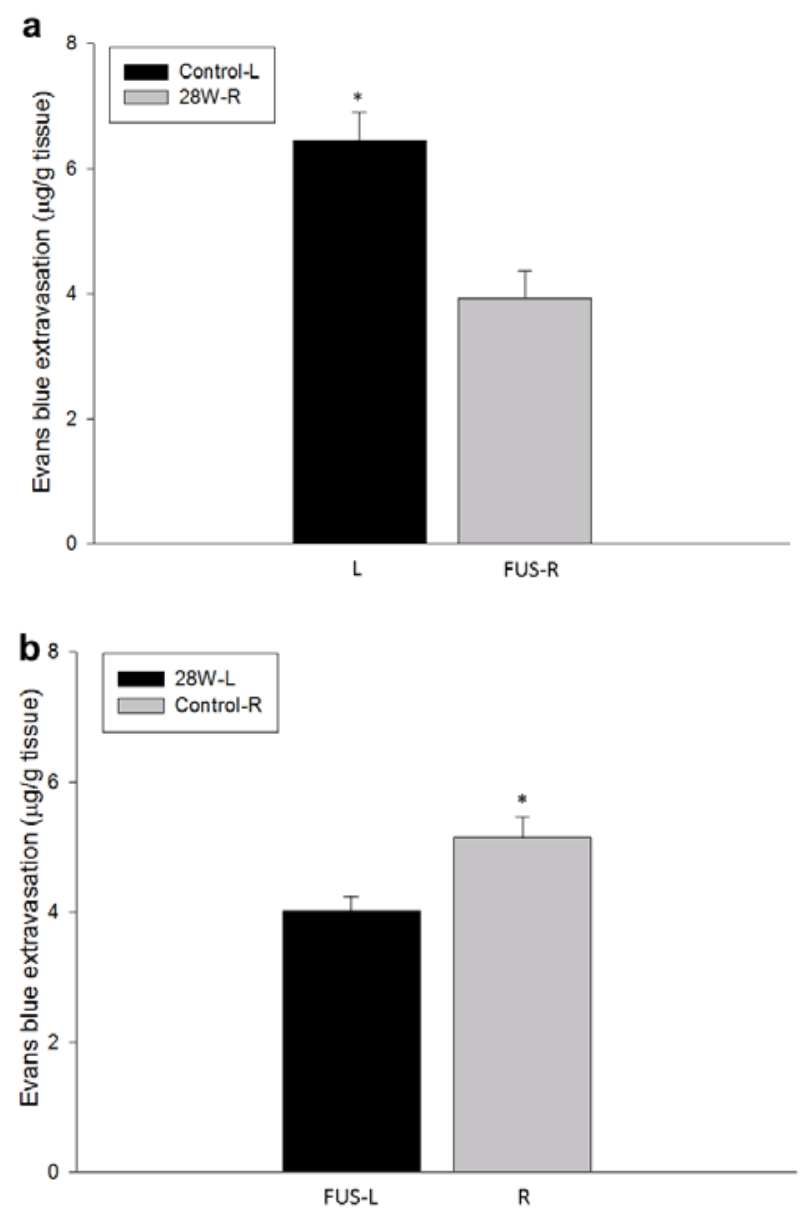

Figure 3. EB extravasation for the brain sonicated at an acoustic power of $2.86 \mathrm{~W}$ in the right "a” or left " $\mathrm{b}$ ” hemisphere. *Denote significant difference compared with the contralateral normal brain.

\section{DISCUSSIONS}

This study demonstrated that the various acoustic powers of FUS exposure alone that affecting BBB permeability as evaluated by EB accumulation. BBB permeability can be enhanced by FUS exposure alone and the efficiency is dependent on the acoustic power of sonication.

Many methods have been created to overcome BBB while chemotherapy is needed, such as chemical modification of drugs, and direct injection through a catheter [10,11]. Recently, FUS with MBs has been developed as a promising tool for target drug delivery in the brain because BBB can be locally and noninvasively disrupted by this technology. One major shortcoming of FUS with MBs is that hemorrhage or brain damage is usually produced by inertial cavitation when the ultrasound parameters and the dose of MBs are not optimized. So far, the behaviors of MBs after sonication are still unable to approximately predict by mathematical model. Therefore, safety concern is the key factor for this technology in the clinical application.

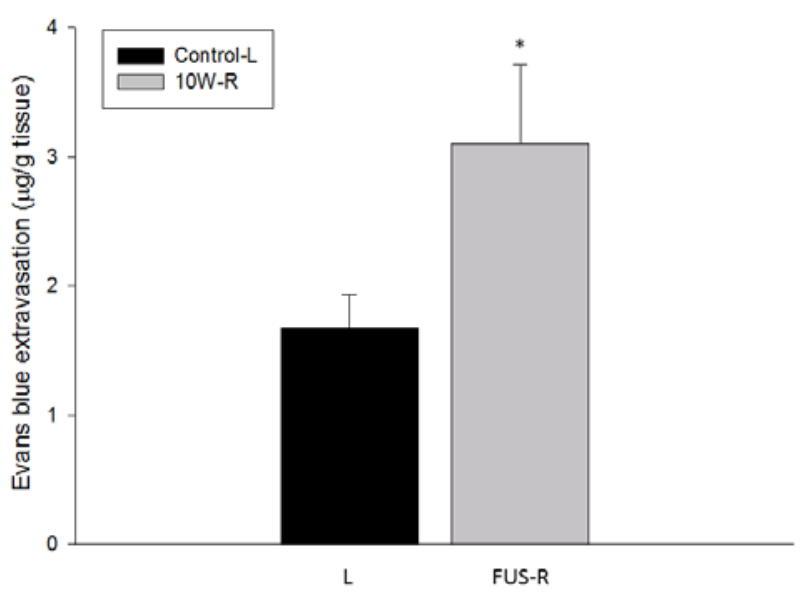

Figure 4. EB extravasation for the brain sonicated with an acoustic power of $1.01 \mathrm{~W}$ in the right hemisphere brain. *Denote significant difference compared with the contralateral normal brain.

One study demonstrated BBB disruption was produced in the thermal lesions after sonication alone, but it has always been associated with tissue damage [12]. Here, we proposed that BBB disruption can be induced by pulsed FUS in the absence of MBs. Figure 3 showed that EB extravasation did not increase in the sonicated brain when sonication was applied at a higher acoustic power of $2.86 \mathrm{~W}$. On the contrary, the permeability of BBB in the contralateral brain significantly increased due to the reflecting ultrasound wave from the bottom skull. Figure 4 showed that EB significantly increased in the sonicated brain when a lower acoustic power of $1.01 \mathrm{~W}$ was applied. The results suggest that the degree of BBB disruption induced by sonication alone was not proportional to the acoustic power. Thus, the degree of localization of BBB disruption can be enhanced by FUS exposure alone at an appropriate acoustic power. The increases in the BBB permeability reported here are important for the safe delivery of drugs to the brain. Further investigations are necessary for optimal ultrasound parameters and histological examinations.

\section{ACKNOWLEDGEMENTS}

This study was supported by grants from the National Science Council of Taiwan (no. NSC 101-2320-B-010-036-MY3, NSC 102-2221-E010-005-MY3), Cheng Hsin General Hospital Foundation (no. 102F218C11 and 101F195CY18).

\section{REFERENCES}

[1] Abbott, N.J. and Romero, I.A. (1996) Transporting therapeutics across the blood-brain barrier. Trends in Molecular Medicine, 2, 106-113. http://dx.doi.org/10.1016/1357-4310(96)88720-X

[2] Yang, F.Y., Lin, Y.S., Kang, K.H. and Chao, T.K. (2011) 
Reversible blood-brain barrier disruption by repeated transcranial focused ultrasound allows enhanced extravasation. Journal of Controlled Release, 150, 111-116. http://dx.doi.org/10.1016/j.jconrel.2010.10.038

[3] Yang, F.Y., Wang, H.E., Lin, G.L., Teng, M.C., Lin, H.H., Wong, T.T. and Liu, R.S. (2011) Micro-SPECT/ CT-based pharmacokinetic analysis of 99mTc-diethylenetriaminepentaacetic acid in rats with blood-brain barrier disruption induced by focused ultrasound. Journal of Nuclear Medicine: Official Publication, Society of Nuclear Medicine, 52, 478-484.

[4] Yang, F.Y., Wong, T.T., Teng, M.C., Liu, R.S., Lu, M., Liang, H.F. and Wei, M.C. (2012) Focused ultrasound and interleukin-4 receptor-targeted liposomal doxorubicin for enhanced targeted drug delivery and antitumor effect in glioblastoma multiforme. Journal of Controlled Release, 160, 652-658. http://dx.doi.org/10.1016/j.jconrel.2012.02.023

[5] Hynynen, K., McDannold, N., Sheikov, N.A., Jolesz, F.A., Vykhodtseva, N. (2005) Local and reversible bloodbrain barrier disruption by noninvasive focused ultrasound at frequencies suitable for trans-skull sonications. Neuroimage, 24, 12-20. http://dx.doi.org/10.1016/j.neuroimage.2004.06.046

[6] Yang, F.Y. and Lee, P.Y. (2012) Efficiency of drug delivery enhanced by acoustic pressure during blood-brain barrier disruption induced by focused ultrasound, International Journal of Nanomedicine, 7, 2573-2582. http://dx.doi.org/10.2147/IJN.S31675

[7] Hynynen, K. (2008) Ultrasound for drug and gene deli- very to the brain. Advanced Drug Delivery Reviews, 60, 1209-1217. http://dx.doi.org/10.1016/j.addr.2008.03.010

[8] Yang, F.Y., Wang, H.E., Lin, G.L., Lin, H.H. and Wong, T.T. (2012) Evaluation of the increase in permeability of the blood-brain barrier during tumor progression after pulsed focused ultrasound. International Journal of $\mathrm{Na}$ nomedicine, 7, 723-730. http://dx.doi.org/10.2147/IJN.S28503

[9] Yang, F.Y., Chen, C.C., Kao, Y.H., Chen, C.L., Ko, C.E., Horng, S.C. and Chen, R.C. (2013) Evaluation of dose distribution of molecular delivery after blood-brain barrier disruption by focused ultrasound with treatment planning. Ultrasound in Medicine \& Biology, 39, 620-627. http://dx.doi.org/10.1016/j.ultrasmedbio.2012.11.027

[10] Kroll, R.A. and Neuwelt, E.A. (1998) Outwitting the blood-brain barrier for therapeutic purposes: Osmotic opening and other means. Neurosurgery, 42, 1083-1099; discussion, 1099-1100. http://dx.doi.org/10.1097/00006123-199805000-00082

[11] Pardridge, W.M. (2002) Drug and gene delivery to the brain: The vascular route. Neuron, 36, 555-558. http://dx.doi.org/10.1016/S0896-6273(02)01054-1

[12] McDannold, N., Vykhodtseva, N., Jolesz, F.A. and Hynynen, K. (2004) MRI investigation of the threshold for thermally induced blood-brain barrier disruption and brain tissue damage in the rabbit brain. Magnetic Resonance in Medicine: Official Journal of the Society of Magnetic Resonance in Medicine/Society of Magnetic Resonance in Medicine, 51, 913-923. 